\title{
Towards Information Customization and Interoperability in Food Chains
}

\author{
Kai Mertins, Frank-Walter Jaekel, and Quan Deng \\ Fraunhofer Institute Production Systems and Design Technology, \\ Pascalstr. 8-9, D-10587 Berlin, Germany \\ \{Kai.Mertins, Frank-walter.Jaekel, Quan. Deng\} @ipk.fraunhofer.de
}

\begin{abstract}
Food supply chain networks are characterized by clouds of various partners providing required products or services to meet customer demands. The coordination of the SMEs (small and medium-sized enterprise) is a major challenge that food chain networks are facing. Delivering the right information to partners and providing semantic interoperability play key roles in effective coordination. This paper proposes an efficient framework to help these SMEs to easily negotiate and shape the profiles of the traceability information products. It tries to involve various tools to model the contexts of SMEs, and then adopt the concepts of mass customization to suggest optimal profiles for the desired information. In supply chain level, agents and governance boarders are supposed to negotiate and determine the profiles of needed information products. The customized information profiles are then employed to map and integrate various information sources to produce the required information products.
\end{abstract}

Keywords: food supply chain network, cloud enterprise, traceability, information product, semantic interoperability, ontology, mass customization, Constraint satisfaction, quality management.

\section{Introduction}

Food supply chain networks are characterized by clouds of various partners providing required products or services to meet customer demands. Most of the partners in food chain networks are SMEs [1]. With limited resources and knowledge, these SMEs usually have problems to adapt dynamic changes in environment. With the equipment of cloud computing, as shown in Fig. 1, SMEs have chances to collaborate with a large number of other partners and services. The various companies distributed among different countries could dynamically choose the partners or services to meet their particular demands. Meanwhile, they have to adapt themselves to meet their own specific contexts, objectives as well as the requirements from other partners. Considering traceability in food chain networks, many SMEs do not have precise knowledge on the required traceability information that best satisfy their own contexts. For the cooperation with other partners in the cloud environment, it is difficult for them to negotiate and then determine the profiles of their desired/exchanged information products.

The original version of this chapter was revised: The copyright line was incorrect. This has been corrected. The Erratum to this chapter is available at DOI: 10.1007/978-3-642-33068-1_20

M. van Sinderen et al. (Eds.): IWEI 2012, LNBIP 122, pp. 92-103, 2012.

() IFIP International Federation for Information Processing 2012 
The SMEs in the clouds of food chain networks are supposed to work collaboratively to provide high-quality food to customers. However, in the last few years, food-safety issues were frequently discussed, such as avian flu, EHEC foodsafety crisis. Meanwhile, the clear awareness of sustainability and healthy regarding food products is increasing importance. Traceability which is usually referred as following the movement of entities throughout a whole supply chain is often taken as an essential tool to increase food quality [2]. Not only movement information, but also much other information related to food products, such as animal warfare, GMO (genetically modified organisms), impact of ingredients to customer's health (e.g. too much sugar in food is not healthy for a patient with diabetes), could be kept as traceability information. The collected traceability information is possible to be used for addressing un-safe food products in case of incidents as well as verification of food quality with respect to safety, healthy and sustainability [3]. There are many other benefits related to traceability information, for instance, increasing transparency, increasing customers' satisfactions, verification of regulation compliance, etc. But setting up associated systems as well as gathering, processing, storing and sharing of the information is costly. For efficient coordination of the SMEs with respect to traceability, delivering the right information to partners and providing semantic interoperability play key roles. For instance, suppose information regarding GMO is critical important for a specific customer group, then all partners in the supply chain would be required to provide and exchange GMO-related information. However, for other customers, GMO information could be ignored to save cost. Customization of the information profiles which are expressed in ontologies would help SMEs to specify what information should be gathered/delivered with respect to their own contexts, objectives and constraints. And the customized information profiles then provide guidelines for information collection. However, to the best knowledge of the authors, currently there is no suitable framework available to help the users in SMEs to easily negotiate and get the food traceability information related ontologies that could best satisfy their particular business strategies, contexts, objectives and constraints.

With respect to delivering the right information to partners and providing semantic interoperability in food chain networks, some requisites should be satisfied by a proposed framework:

- Framework should provide tools and methodologies to help SMEs specify their own contexts, objectives and constraints with respect to traceability.

- Based on the provided information from SMEs, the framework should be able to propose optimal information profiles (expressed in ontologies) which could best satisfy SMEs' contexts, objectives and constraints.

- Distinctive information profiles should be provided for diversity products under different environments (e.g. different customer demands).

- In case of dynamic changes in business environment, SMEs should be able to easily get their own suitable information profiles.

- Semantic interoperability between information products should be guaranteed, so that SMEs could freely exchange information with other potential partners. 
- Partners in the same supply chain should be coordinated to comply with a common information profiles to support all necessary traceability scenarios. For instance, if GMO information at broiler rearing stage is required in a supply chain, then all broiler farms in the supply chain are expected to provide GMO information.

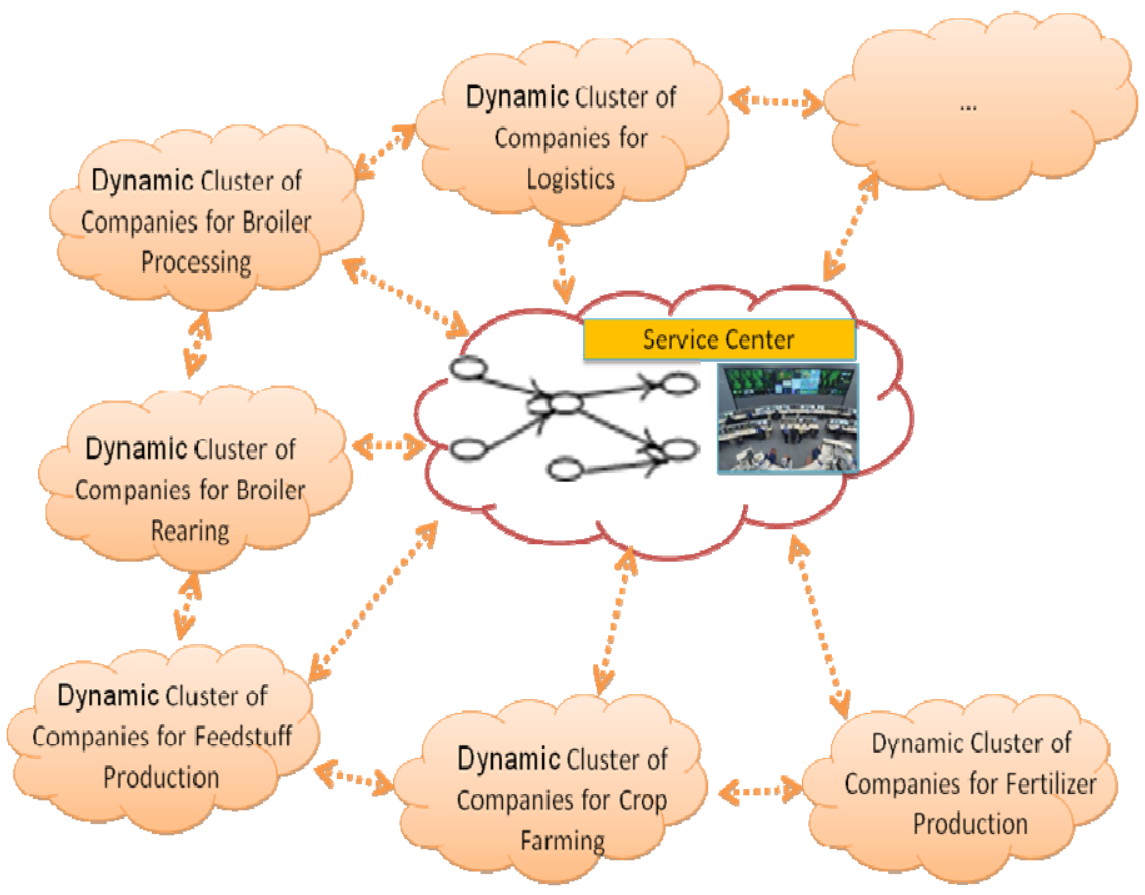

Fig. 1. Sample Cloud Environment for Broiler Supply Chains

The following section provides a review on related areas. And then, section 3 briefly described the proposed approaches. Finally, conclusion and future work to be done are presented.

\section{Related Works}

Traceability was first clearly described by Kim in the context of the TOVE (Toronto Virtual Enterprise) project [4]. Since then, numerous researches were conducted in this area. Traceability systems evolving from centralized to distributed "one step up, one step down", are becoming more distributed, interoperable and intelligent. More and more technologies, e.g. RFID (Radio), Frequency Identification), SOA (Service Oriented Architecture), web service, TraceCoreXML, ebXML (Electronic Business using extensible Markup Language), EDI (Electronic Data Interchange) etc. are used to facilitate integration and interoperability in food traceability. In the process of setting up an effective traceability system, one of the most important things is to 
determine the information to be recorded [5]. However, Partners are usually not sure, what the optimal traceability level is and what the exactly data that should be gathered is. Depends on traceability objectives, product characteristics, production processes, consumers' expectations and regulations, etc., the needed traceability data elements are different from one to another. There is no common accepted standard regarding the needed traceability data elements, and there is no single best way to introduce traceability because of the differences in product attributes and motivation of traceability etc. [6]. Partners usually collect only limited traceability information for regulation compliance. But for many other purposes, e.g. sustainability verification, the information is usually not sufficient enough to bring maximum benefit. The SMEs are difficult to be coordinated to provide all necessary information for various traceability scenarios.

To standardize the traceability data elements, some reference data models on traceability, such as the model from von Drop [7], and Bechini et al [8], were proposed. But they seem to be in a high general level, which cannot easily be customized or configured for partners in a food chain network under a specific environment. The detailed needed data elements cannot be easily derived from the models. Many constraints such as customers' expectations on traceability, regulations are not considered. Partners worldwide with varying regulations and business restrictions, have different markets and traceability expectations from consumers. Some consumers with some diseases, like diabetes or obesity, would have more interest in the traceability information related to nutrition and healthy. Others who concern more on the sustainability aspects (e.g. carbon emission) would be more interested in traceability information regarding sustainability. Besides, foods supply chain networks are becoming more demand driven and dynamical [9]. These dynamics in networks imply corresponding changes in the traceability needs, as well as the desired traceability data elements.

Information customization is to tailor information to meet special customer demands [10]. It is usually helpful to personalize information and avoid information overload. Abidi and Han presented an information customization framework which applied constraint satisfaction methods to select the hypermedia documents that conform to the user model [11]. Related to identifying the to be recorded traceability information, Diogo M. Souza-Monteiro et al. propose a framework based on vertical control and agency theory to model three dimensions of traceability systems: depth, breadth, and precision [12]. They model and analysis voluntary traceability system in a supply chain producing multi-ingredient foods from the point view of economic implications. However, their assumptions simplified the real supply chain networks, and many other factors besides economic implications such as production process, traceability motivations, products' characteristics, and regulations etc., which influence the determination of the information profiles, are not considered. For the products/ingredients that result more risk than others, more detailed information should be recorded. Thakur et al. present a soybean value chain and model the information that should be captured by three links in the chain [13]. On the basis of process models, they specify the data elements that should be included in the traceability information. Based on the information profiles, related information is then gathered, integrated to produce needed traceability information products. For 
information integration and semantic interoperability, the information profiles of SMEs are expressed as local ontologies that represent SMEs' own perspectives on traceability. Then the problem could be transformed into customization of optimal local ontologies that satisfy various constraints and objectives.

Ontology construction is one of the central research issues in the ontology area. There are lots of tools available for ontologies development, such as Protégé [14]. Regarding methodologies for ontology construction, it could trace back to the Cyc Project, in which the experience in the Cyc ontology development process is recorded [15]. Some years later, in the enterprise modelling domain, the experience in development TOVE ontology [4] is gathered. And then many other methodologies such as METHONTOLOGY [16], On-To-Knowledge [17] are presented. To support collaborative and distributed ontology construction, some methodologies like DOGMA [18], DILIGENT [19], Onto-Agent [20] were presented. In DOGMA and Onto-Agent methodologies, ontology resources are explicitly decomposed into ontology bases and ontological commitment. In ontology base, intuitive concepts and relationships between concepts within a domain are kept. In the ontology commitment layer, a set of constraints and rules are contained to allow agents to specify commitment ontology from their own point of view. In DILIGENT methodology, an initial shared ontology is first constructed, and then users could locally adapt the shared ontology for their own purposes. However, in the DOGMA, DILIGENT and Onto-Agent methodologies, users should have enough knowledge on ontology and know clearly about their desired ontology in order to reuse and construct their own ontologies. Farqahar et al. introduced Ontolingua Server for Collaborative Ontology Construction [21]. It supports ontologies reuse through an ontologies library, although the supports for ontology development in a distributed and collaborative environment are still limited [22]. To provide better supports for collaborative and argumentative ontology development as well as on the building of ontology networks, $\mathrm{NeOn}$ Methodology was then proposed [22] [23]. It considers the reuse and possible subsequent reengineering of knowledge resources as one of the key aspects.

Ontology selection is the process to identify one or more ontologies or ontology modules that satisfy certain criteria [24]. In the process, Ontologies could be searched based on specific keywords, logic query, ontology or some more complex query mechanism [24]. Some ontology search engines were presented in last few years, such as Swoogle [25] and OntoSelect [26]. Some of them like OntoSelect are ontology libraries that offer the ability of ontology selection. The SAIQL (Schema And Instance Query Language for OWL DL) proposed in NeOn project, is a sample language well suited for to extract a domain oriented sub-ontology [27].On the ontology selection, sub-ontology extraction and ontology pruning process, users usually need to input the related concepts either by manual input or extraction from other documents/knowledge sources. Some approaches such as TEXT-TO-ONTO [28] generate ontology based on the concepts discovered from texts or other resources. Lonsdale et al. take natural language (NL) documents and source ontologies as input, and then select/discovery related concepts etc. from source ontologies on the basis of descriptions in NL documents [29]. The main focus in these approaches are discovery/select concepts etc. based on documents for ontology generation, but understanding the contexts of SMEs 
and then proposing optimal ontologies to best satisfy SMEs' contexts is not the advantage of their approaches.

Although in the construction process, lots of the reuse processes are supported by semantic web technologies like ontology searching, selecting, merging, evaluation, but these technologies are still immature. Most of the processes still need lots of manual interventions. Moreover, they are mostly based on a hidden assumption that users know which the right ontologies considering their particular contexts are, and then users are able to understand, select and reuse ontologies for their own ontology construction. However, when the ontologies as well as application contexts and constraints become increasing complex, for instance, in the traceability context, users (e.g. users in SMEs) usually have no clear ideas about what the optimal traceability level and optimal information profiles (optimal ontologies) for them are. They do not know exactly whether a concept or property should be encompassed. The users in SMEs do have much knowledge on their own process models, motivations of traceability and complied regulations etc., but have little knowledge regarding ontology construction, as a result, the existing approaches for ontology construction would be unsuitable or a great challenge for them.

The term of Mass customization coined by Davis [30] was defined as "producing goods and services to meet individual customer's needs with near mass production efficiency" [31]. As the definition states, the goals of mass customization are to best server various customers' needs while at the same time maintain high efficiency. Production configuration for generating a product variant for a rapid response of customers' individual demands is one of the key approaches to enable mass customization. Considering the diversities in business environments and dynamics in traceability needs, the concept of mass customization used in manufacturing is possible to be applied in the customization of "local ontologies" (information profile) for traceability in SMEs.

\section{Proposed Approaches}

In order to answer aforementioned challenges, a framework which adopts the concepts of mass customization to shape the right information profiles will be presented. The core components for ontology customization in the framework are shown in the Fig. 2.

The service side provides toolkits for domain experts to maintain generic ontology structures, modularized reference ontologies and associated knowledge bases. In the repositories, the ontology modules are kept and the interoperability between ontology modules is pre-analyzed. For reusing and mass customization, related context information is attached to the ontology modules. The context information of ontology modules will be then associated with models of SMEs to help to select the right modules. In the knowledge base, the knowledge related to food traceability as well as the knowledge related to ontology customization like generic ontology structure are kept. Meanwhile, toolkits for SMEs are provided to support them model their own contexts, constraints etc. With the help of the toolkits, the factors like regulations, customer requirements and process models, etc., which would drive the selection of traceability data elements and customization of ontologies, could be clearly modelled. 


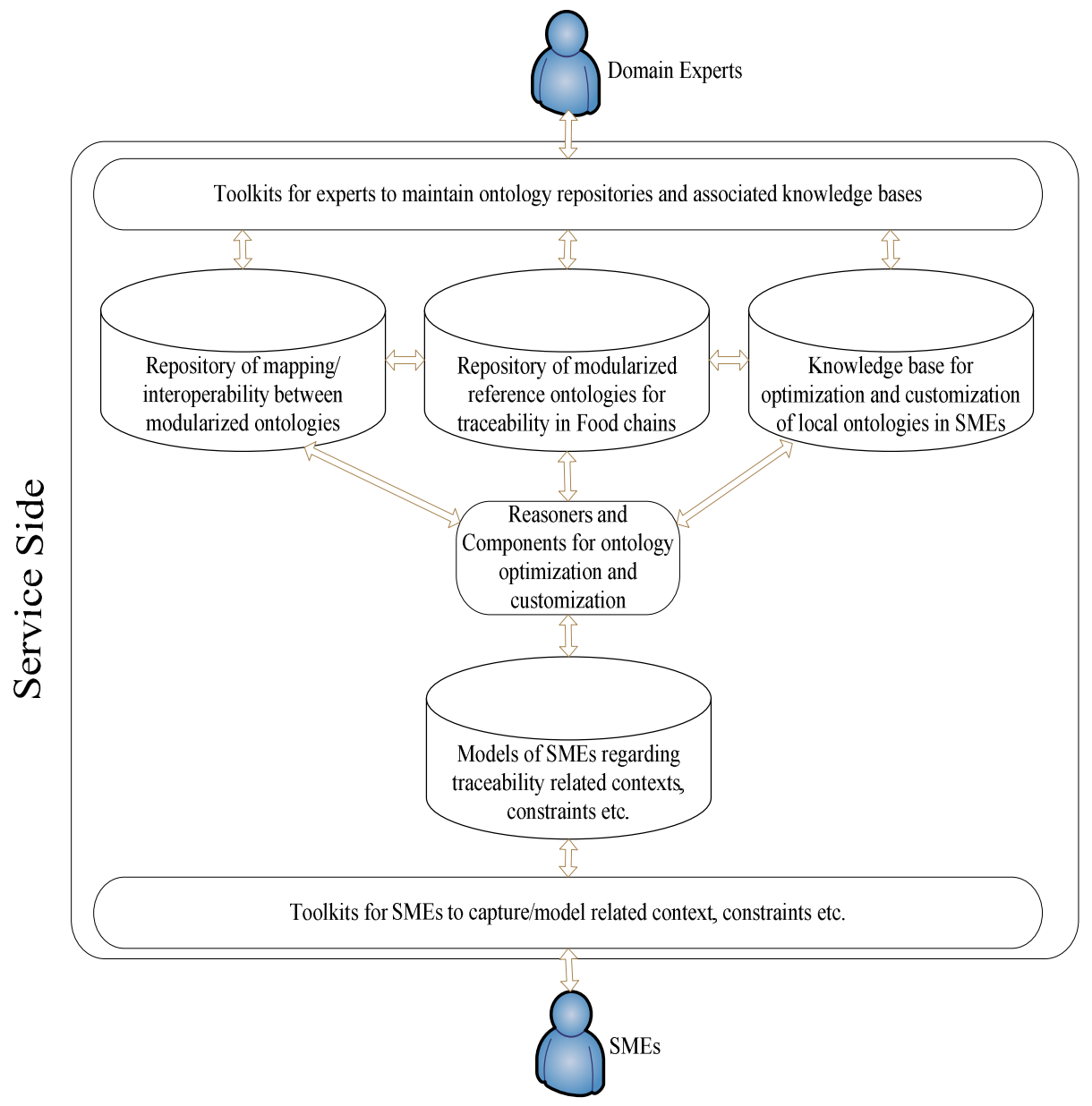

Fig. 2. Basic Structure in Framework for Ontology Customization

For instance, the following or toolkits could be included:

- Toolkits for business process model. Business process models represent processes, resources, products, etc. of an enterprise. Based on the process models, the potential information capture points as well as related costs and constraints are identified. The toolkits could be based on available tools like $\mathrm{MO}^{2} \mathrm{GO}[32]$.

- Toolkits for capturing customer requirements. In order to satisfy customer needs, requirements from customers should be captured and reflect in the information profiles. For example, if health impact of the foods with respect to special customer groups is critical importance, then collection, processing and evaluation health associated information (e.g. nutrition) would be in a relative high priority. Some toolkits from quality management areas like Quality Function Deployment (QFD) are possible to be equipped in the framework. 
After the reference modularized ontologies, knowledge bases and the environments of SMEs etc. are specified within the framework, the methodologies used in mass customization, e.g. constraint satisfaction approach, will be employed to propose optimal local ontologies. In the customization process, ontology modules which satisfy the requirements and targets of SMEs' contexts are selected and then merged. For example, if the models of a SME show that sustainability information of a food product is demanded only by a few consumers and is costly to be gathered, then the ontology modules related to sustainability information would not be selected. In the local contextualized ontologies, the data elements/concepts which the SMEs really interested in are displayed. Based on customized ontologies, information dispersed in many other systems is then integrated to produce the needed traceability information products, as shown in Fig. 3. Besides, it provides guidance for the SMEs to collect and prepare the required but missed data elements. Since the customized local ontologies of SMEs are generated on the basis of the repository of modularized ontologies, the semantic interoperability between information products is then supported by the pre-specified interoperability of the reference modularized ontologies.

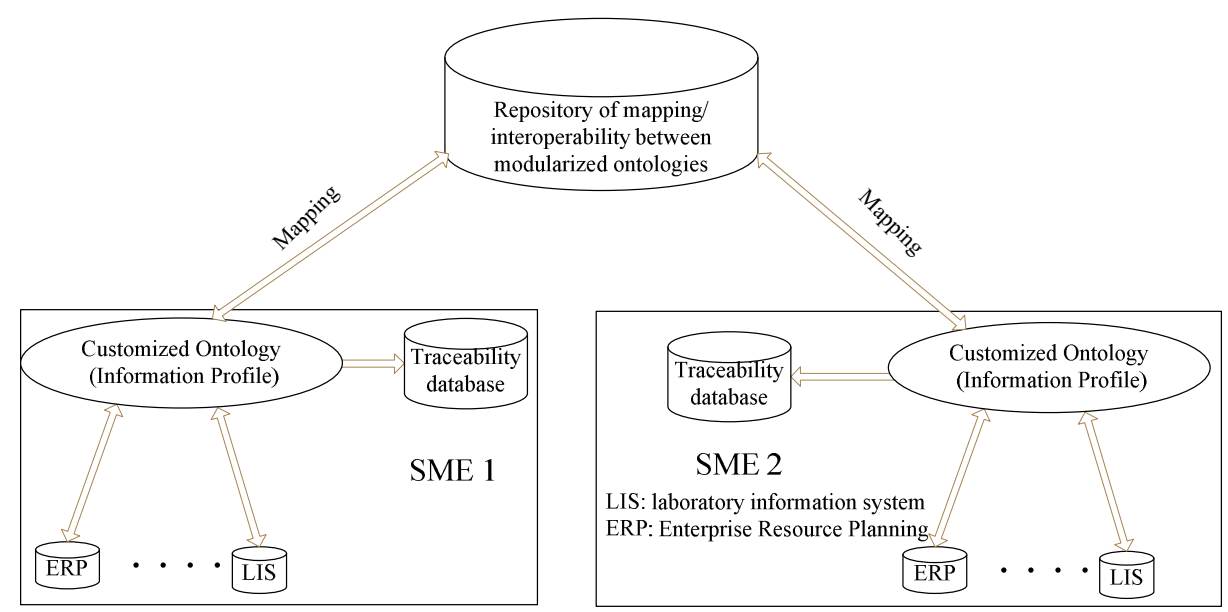

Fig. 3. Interoperability between SMEs

To ensure that the required traceability data supporting necessary scenarios are available within all partners of a supply chain, the partners producing the same ingredients in the same supply chain should negotiate to have a customized common shared ontology. In the ontology, the traceability information that needs to be exchanged in the supply chain is explicit expressed. Fig. 4 shows a mechanism for the coordination and determination of the common shared ontology in a sample supply chain. For each specific kind of partners (e.g. broiler farm), a high-level agent is initialized. The high-level agent will first coordinate with its sub-level agents i.e. agents of SMEs, as well as with other high-level agents to get necessary information. The information is then transformed and used as input for the aforementioned 
ontology customization approach to suggest common shared ontologies for the supply chain. At last, a governance boarder will make some modifications and determine the customized common shared ontology in the supply chain. The common shared ontologies describe the traceability information that will be exchanged and provided by partners. When there are some dynamic changes in business environment (e.g. Changes in partnership or customer demands), the ontologies could be easily recustomized to adapt to the new environment. For instance, imagine sustainability related information is currently missed in a food supply chain, but when this kind of information is required by regulations, and at the same time their target consumers are willing to pay for it, then the common shared ontology as well as local ontologies of the partners need to be quickly re-customized to encompass the sustainability related data elements/concepts.

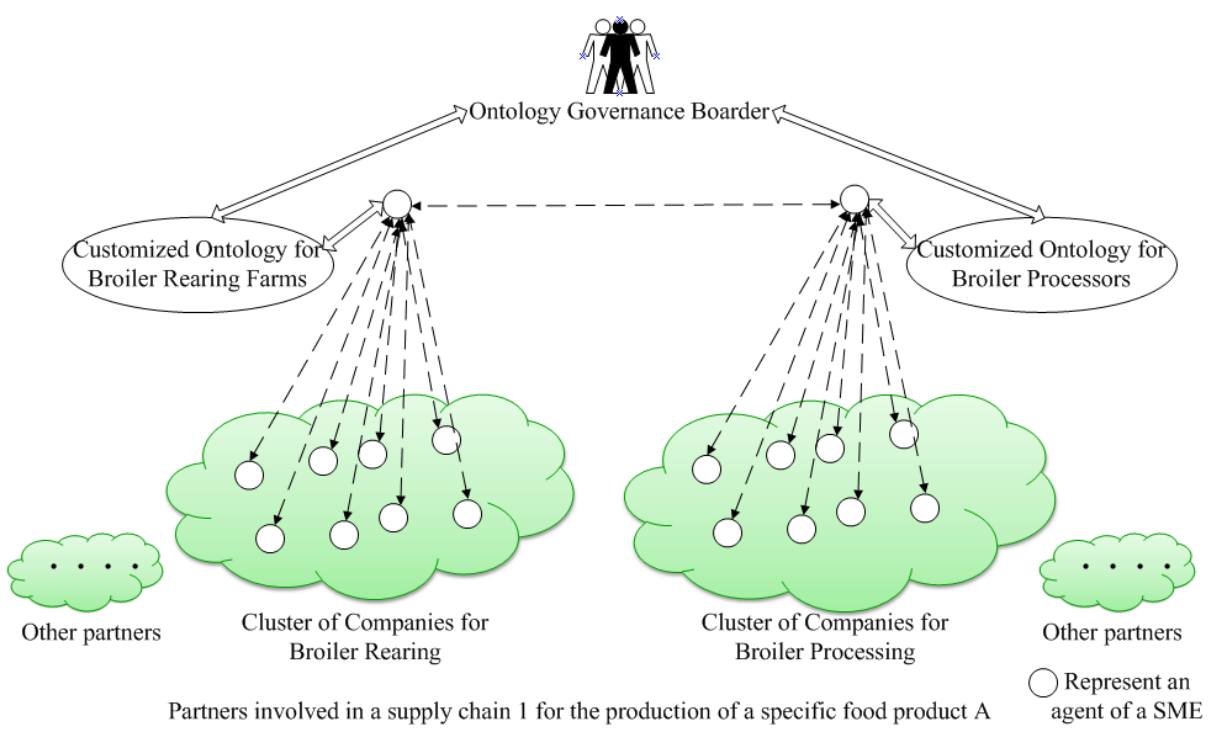

Fig. 4. Coordination Mechanism for Determination of Common Ontologies in a Sample Broiler Supply Chain

\section{Conclusions}

Identifying the list of data elements to be recorded is significant important for an efficient supply chain traceability systems. Depending on product characteristics, process model, customers' demands, motivation of traceability etc., the optimal information profiles which specify the needed traceability information are usually different from one to another. For information integration and semantic interoperability, the information profiles are expressed as local ontologies that represent SMEs' own perspectives on traceability information. Considering the dynamics in business environments and diversities in traceability needs, the paper adopts the concept of mass customization into the customization of these local 
ontologies /information profiles. An efficient framework to help these SMEs to negotiate and shape the right traceability information products is briefly presented. It involves the toolkits to help SMEs to model traceability relevance factors, such as business process models, customer demands, etc. With the pre-specified interoperability between modularized reference ontologies, semantic interoperability of the traceability information between various SMEs is also guaranteed. To ensure that the traceability data required to support necessary traceability scenarios are available within all partners of a supply chain, multi-agents and ontology governance boarder are introduced in the coordination mechanism. In traceability areas, this paper is supposed to provide a methodology for the determination of the to be recorded traceability information. In ontology construction area, it tries to adopt the concepts of mass customization to help the users who do not know clearly what right ontology for their purposes is and the ones who are not familiar with ontology technologies to easily get the customized ontologies that could satisfy their particular contexts. For the collaboration of the SMEs in food chain networks, a coordination mechanism is simply introduced.

In future work, we will start detailing the components, associated toolkits and building the framework described in the paper. Definitely, there are many challenges to build the system and run successfully in food industry. Because the ontologies in SMEs are customized through reusing modularized ontologies, the quality and granularity of ontology modules will certainly affect the quality of customized ontologies. The context information of ontology modules should be carefully designed, so that the modules could be correctly reused. With respect to context models of SMEs, some issues like how to model the driving factors and then use them effectively for the selection of traceability data elements as well as customization of related ontologies still need to be further detailed. For the negotiation of common shared ontologies, the cooperation and communication between agents will be further specified. And then the approach is expected to be experimented in food industry to evaluate how well it works in real business scenarios.

\section{References}

1. Food industry, EU food market overview, http://ec.europa.eu/enterprise/ sectors/food/eu-market/index_en.htm

2. ISO: ISO 22005:2007, Traceability in the feed and food chain - General principles and basic requirements for system design and implementation, 1st edn., Switzerland (2007)

3. Hobbs, J.E.: Information asymmetry and the role of traceability systems. Agribusiness 20, 397-415 (2004)

4. Kim, H.M., Fox, M.S., Gruninger, M.: An ontology of quality for enterprise modeling. In: Proceedings 4th IEEE Workshop on Enabling Technologies: Infrastructure for Collaborative Enterprises (WET ICE 1995), pp. 105-116 (1995)

5. Regattieri, A., Gamberi, M., Manzini, R.: Traceability of food products: general framework and experimental evidence. Journal of Food Engineering 81, 347-356 (2007)

6. Golan, E., Krissoff, B., Kuchler, F., Calvin, L., Nelson, K., Price, G.: Traceability in the U. S. Food Supply: Economic Theory and Industry Studies Library Cataloging Record. Economic Theory (830) (2004) 
7. van Dorp, C.: Reference-data modelling for tracking and tracing. Wageningen University (2004)

8. Bechini, A., Cimino, M.G.C.A., Lazzerini, B., Marcelloni, F., Tomasi, A., Elettronica, I.: A General Framework for Food Traceability. In: Proceedings of the 2005 Symposium on Applications and the Internet Workshops, pp. 366-369 (2005)

9. Verdouw, C.: Business process modelling in demand-driven agri-food supply chains: a reference framework. Wageningen University (2010)

10. Berleant, D., Berghel, H.: Customizing Information: Part I - IEEE Computer, 27(9), 96-98 (1994); Part II - IEEE Computer, 27(10), 76-78 (1994)

11. Abidi, S.S.R., Chong, Y.: An adaptive hypermedia system for information customization via content adaptation. IADIS Intl. Journal of WWW/Internet 2(1), 79-94 (2004)

12. Souza-monteiro, D.M., Caswell, J.A.: The Economics of Voluntary Traceability in MultiIngredient Food Chains. Agribusiness 26(1), 122-142 (2010)

13. Thakur, M., Donnelly, K.A.-M.: Modeling traceability information in soybean value chains. Journal of Food Engineering 99(1), 98-105 (2010)

14. The Protégé Ontology Editor and Knowledge Acquisition System, http: / / protege.stanford.edu/

15. Elkan, C.: Building Large Knowledge-Based Systems:Representation and Inference in the Cyc Project book review 1 Comments on the Cyc project. Readings, pp. 1-11 (1989)

16. Ferndndez, M., Gmez-Perez, A., Juristo, N.: METHONTOLOGY: From Ontological Art Towards Ontological Engineering. Assessment, pp. 33-40 (1997)

17. Staab, S., Schnurr, H.-P., Studer, R., Sure, Y.: Knowledge Processes and Ontologies. Knowledge Creation Diffusion Utilization (2000)

18. Jarrar, M., Meersman, R.: Formal Ontology Engineering in the DOGMA Approach. In: Meersman, R., Tari, Z. (eds.) CoopIS 2002, DOA 2002, and ODBASE 2002. LNCS, vol. 2519, pp. 1238-1254. Springer, Heidelberg (2002)

19. Pinto, S., Staab, S., Tempich, C., Sure, Y.: DILIGENT: Towards a fine-grained methodology for Distributed, Loosely-controlled and evolvInG Engineering of oNTologies. In: N.N. (eds.), ECAI. IOS Press (2004)

20. Hadzic, M.: Onto-agent methodology for design of ontology-based multi-agent systems. Computer Systems Science and Engineering 34(3), 358-364 (2008)

21. Farquhar, A., Fikes, R., Rice, J.: The ontolingua server: A tool for collaborative ontology construction. International Journal of Human-Computer Studies (1996)

22. del Carmen Suárez, M., de Baonza, F.: NeOn Methodology for Building Ontology Networks: Specification, Scheduling and Reuse. Doctoral Thesis in Building (2010)

23. NeOn Project, http: / /www. neon-project.org/

24. Sabou, M., Lopez, V., Motta, E., Uren, V.: Ontology Selection: Ontology Evaluation on the Real Semantic Web, Victoria (2006)

25. Ding, L., et al.: Swoogle: a search and metadata engine for the semantic web. In: Proceedings of the Thirteenth ACM International Conference on Information and Knowledge Management, pp. 652-659 (2004)

26. Buitelaar, P., Eigner, T., Declerck, T.: OntoSelect: A Dynamic Ontology Library with Support for Ontology Selection. In: The OntoSelect Ontology Library. Research Management, pp. 3-6 (2003)

27. Kubias, E., Schenk, S., Staab, S., Pan, J.Z.: OWL SAIQL-an OWL DL query language for ontology extraction. In: Proc. of OWLED 2007 (2007)

28. Maedche, E., Staab, S.: The TEXT-TO-ONTO Ontology Learning Environment. In: Software Demonstration at ICCS 2000 - Eight International Conference on Conceptual Structures (2000) 
29. Lonsdalea, D., Embley, D.W., et al.: Reusing Ontologies and Language Components for Ontology Generation. Journal Data \& Knowledge Engineering 69(4), 318-330 (2010)

30. Davis, S.M.: From 'future perfect': Mass customizing. Strategy \& Leadership 17(2), 16-21 (1989)

31. Tseng, J., Jiao, M.M.: Mass customization. In: Handbook of Industrial Engineering, 3rd edn., New York (2001)

32. Mertins, K., Jochem, R.: $\mathrm{MO}^{2}$ GO. In: Handbook on Architectures of Information Systems. Springer, Berlin (1998) 